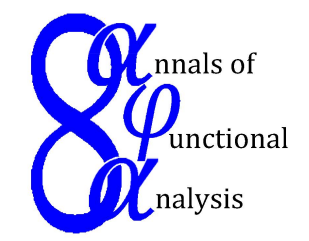

Ann. Funct. Anal. 6 (2015), no. 4, 155-171

http://doi.org/10.15352/afa/06-4-155

ISSN: 2008-8752 (electronic)

http://projecteuclid.org/afa

\title{
WEIGHTED INEQUALITIES FOR A CLASS OF SEMIADDITIVE OPERATORS
}

\author{
RYSKUL OINAROV ${ }^{1}$ AND AIGERIM KALYBAY ${ }^{2 *}$ \\ Communicated by S. Barza
}

Abstract. We find necessary and sufficient conditions for the validity of weighted Hardy-type inequalities for a class of semiadditive operators.

\section{INTRODUCTION}

Let $I=(0, \infty), 0<\theta, q, p \leq \infty$ and $\frac{1}{p}+\frac{1}{p^{\prime}}=1$. Suppose that $w, u$ and $v$ are non-negative functions such that they, together with $v^{1-p^{\prime}}$, are locally integrable on $I$.

We introduce the following operators:

$$
T_{\theta}^{+} f(x)=\left(\int_{0}^{x} w(t)\left|\int_{t}^{x} f(s) d s\right|^{\theta} d t\right)^{\frac{1}{\theta}}, T_{\theta}^{-} f(x)=\left(\int_{x}^{\infty} w(t)\left|\int_{x}^{t} f(s) d s\right|^{\theta} d t\right)^{\frac{1}{\theta}}
$$

The operators $T_{\theta}^{+}$and $T_{\theta}^{-}$are superlinear for $0<\theta<1$ and sublinear for $\theta>1$. These operators become linear for $\theta=1$.

We consider the inequalities:

$$
\left(\int_{0}^{\infty} u(x)\left|T_{\theta}^{ \pm} f(x)\right|^{q} d x\right)^{\frac{1}{q}} \leq C^{ \pm}\left(\int_{0}^{\infty} v(t)|f(t)|^{p} d t\right)^{\frac{1}{p}}
$$

where $C^{ \pm}$are positive constants. Let us notice that the Hardy-type inequality with the operator $T_{\theta}^{+}$is directly equivalent to the inequality with the operator

Date: Received: Jan. 13, 2015; Revised: Mar. 10, 2015; Accepted: Mar. 31, 2015.

* Corresponding author.

2010 Mathematics Subject Classification. Primary 26D10; Secondary 46E30.

Key words and phrases. Hardy inequality, semiadditive operator, weight estimate. 
$T_{\theta}^{-}$, via a simple change of variable in the integrals. Moreover, it suffices to study (1.1) for $f \geq 0$.

Let

$$
\Delta_{\theta}^{+} g(x)=\left(\int_{0}^{x} w(t)|g(x)-g(t)|^{\theta} d t\right)^{\frac{1}{\theta}}, \Delta_{\theta}^{-} g(x)=\left(\int_{x}^{\infty} w(t)|g(t)-g(x)|^{\theta} d t\right)^{\frac{1}{\theta}}
$$

be the $\theta$-mean deviations with the weight $w$ of the value of a function $g$ from $g(x)$ on the intervals $(0, x)$ and $(x, \infty)$, respectively. Then inequality (1.1) is equivalent to the following inequality:

$$
\left(\int_{0}^{\infty} u(x)\left|\Delta_{\theta}^{ \pm} g(x)\right|^{q} d x\right)^{\frac{1}{q}} \leq C^{ \pm}\left(\int_{0}^{\infty} v(t)\left|g^{\prime}(t)\right|^{p} d t\right)^{\frac{1}{p}} .
$$

Inequality (1.1) was investigated in [5], where necessary and sufficient conditions for its validity were found for $1 \leq p \leq q<\infty$ and $0<\theta<\infty$.

In this work we study the case $0<q<p, p \geq 1$ and $0<\theta<\infty$. Here we prove the sufficiency part of the provided case and the general $v$. Necessity is derived for the case $0<\theta, q<\infty, \max \{\theta, q\}<p, p>1$ and the general $v$. Moreover, necessity is also derived for the case $\max \{\theta, q\}<1=p$ and $v \equiv 1$.

For $(\alpha, \beta) \subset I$ we assume

$$
\begin{gathered}
A^{+}(\alpha, \beta)=\sup _{\alpha<x<\beta}\left(\int_{\alpha}^{x} w(t) d t\right)^{\frac{1}{\theta}}\left(\int_{x}^{\beta} v^{1-p^{\prime}}(s) d s\right)^{\frac{1}{p^{\prime}}}, \\
A^{-}(\alpha, \beta)=\sup _{\alpha<x<\beta}\left(\int_{x}^{\beta} w(t) d t\right)^{\frac{1}{\theta}}\left(\int_{\alpha}^{x} v^{1-p^{\prime}}(s) d s\right)^{\frac{1}{p^{\prime}}}, \\
B^{+}(\alpha, \beta)=\left(\int_{\alpha}^{\beta} w(t)\left(\int_{\alpha}^{t} w(s) d s\right)^{\frac{\theta}{p-\theta}}\left(\int_{t}^{\beta} v^{1-p^{\prime}}(s) d s\right)^{\frac{\theta(p-1)}{p-\theta}} d t\right)^{\frac{p-\theta}{p \theta}}, \\
B^{-}(\alpha, \beta)=\left(\int_{\alpha}^{\beta} w(t)\left(\int_{t}^{\beta} w(s) d s\right)^{\frac{\theta}{p-\theta}}\left(\int_{\alpha}^{t} v^{1-p^{\prime}}(s) d s\right)^{\frac{\theta(p-1)}{p-\theta}} d t\right)^{\frac{p-\theta}{p \theta}}, \\
D^{+}(\alpha, \beta)=\left(\int_{\alpha}^{\beta} w(t)\left(\int_{\alpha}^{t} w(s) d s\right)^{\frac{\theta}{1-\theta}}(\underline{v}(t, \beta))^{\frac{\theta}{\theta-1}} d t\right)^{\frac{1-\theta}{\theta}},
\end{gathered}
$$




$$
D^{-}(\alpha, \beta)=\left(\int_{\alpha}^{\beta} w(t)\left(\int_{t}^{\beta} w(s) d s\right)^{\frac{\theta}{1-\theta}}(\underline{v}(\alpha, t))^{\frac{\theta}{\theta-1}} d t\right)^{\frac{1-\theta}{\theta}}
$$

where $\underline{v}(\alpha, \beta)=\underset{\alpha<t<\beta}{\operatorname{essinf}} v(t)$.

Let $H^{+}(\alpha, \beta)$ and $H^{-}(\alpha, \beta)$ be the best constants of the following Hardy inequalities

$$
\begin{aligned}
& \left(\int_{\alpha}^{\beta} w(t)\left|\int_{t}^{\beta} f(s) d s\right|^{\theta} d t\right)^{\frac{1}{\theta}} \leq H^{+}(\alpha, \beta)\left(\int_{\alpha}^{\beta} v(t)|f(t)|^{p} d t\right)^{\frac{1}{p}}, \\
& \left(\int_{\alpha}^{\beta} w(t)\left|\int_{\alpha}^{t} f(s) d s\right|^{\theta} d t\right)^{\frac{1}{\theta}} \leq H^{-}(\alpha, \beta)\left(\int_{\alpha}^{\beta} v(t)|f(t)|^{p} d t\right)^{\frac{1}{p}},
\end{aligned}
$$

respectively.

From the results of the works [4] and [8] (see also [5]) for Hardy inequalities we have

Lemma A. (i) If $1 \leq p \leq \theta<\infty$, then

$$
A^{ \pm}(\alpha, \beta) \leq H^{ \pm}(\alpha, \beta) \leq p^{\frac{1}{\theta}}\left(p^{\prime}\right)^{\frac{1}{p^{\prime}}} A^{ \pm}(\alpha, \beta)
$$

(ii) If $0<\theta<p$ and $1<p<\infty$, then

$$
\left(p^{\prime}\right)^{\frac{1}{p^{\prime}}} \theta^{\frac{1}{p}}\left(1-\frac{\theta}{p}\right) B^{ \pm}(\alpha, \beta) \leq H^{ \pm}(\alpha, \beta) \leq\left(\frac{p}{p-\theta}\right)^{\frac{p-\theta}{p \theta}} p^{\frac{1}{p}}\left(p^{\prime}\right)^{\frac{1}{p^{\prime}}} B^{ \pm}(\alpha, \beta) .
$$

(iii) If $0<\theta<1=p$, then

$$
\theta(1-\theta) D^{ \pm}(\alpha, \beta) \leq H^{ \pm}(\alpha, \beta) \leq(1-\theta)^{\frac{1-\theta}{\theta}} D^{ \pm}(\alpha, \beta)
$$

Since the expressions $A^{ \pm}, B^{ \pm}$and $D^{ \pm}$are decreasing in $\alpha$ and increasing in $\beta$, then from $(1.2),(1.3)$ and (1.4) we have that $H^{ \pm}(\alpha, \beta)$ are equivalent to a decreasing function in $\alpha$ and equivalent to a increasing function in $\beta$. It means that for each case (i), (ii) and (iii) there exists a constant $C>0$ depending only on $p$ and $\theta$ such that $H^{ \pm}(\alpha, \beta) \leq C H^{ \pm}\left(\alpha_{1}, \beta_{1}\right)$ holds for $\alpha_{1} \leq \alpha<\beta \leq \beta_{1}$. For example, for the case (i) we have $C=p^{\frac{1}{\theta}}\left(p^{\prime}\right)^{\frac{1}{p^{\prime}}}$.

Denote $A^{+}(0, \beta) \equiv A^{+}(\beta), B^{+}(0, \beta) \equiv B^{+}(\beta), D^{+}(0, \beta) \equiv D^{+}(\beta), H^{+}(0, \beta) \equiv$ $H^{+}(\beta), A^{-}(\alpha, \infty) \equiv A^{-}(\alpha), B^{-}(\alpha, \infty) \equiv B^{-}(\alpha), D^{-}(\alpha, \infty) \equiv D^{-}(\alpha)$ and $H^{-}(\alpha, \infty) \equiv H^{-}(\alpha)$.

In what follows we write $A \ll B$ if $A \leq C B$ with some constant $C>0$ that depends only on $\theta, q$ and $p$. The expression $A \approx B$ means $A \ll B$ and $B \ll A$. 


\section{MAin RESUlts}

Let

$$
\begin{aligned}
& E^{+}=\left(\int_{0}^{\infty} u(x)\left(\int_{x}^{\infty} u(s) d s\right)^{\frac{q}{p-q}}\left(H^{+}(x)\right)^{\frac{p q}{p-q}} d x\right)^{\frac{p-q}{p q}}, \\
& E^{-}=\left(\int_{0}^{\infty} u(x)\left(\int_{0}^{x} u(s) d s\right)^{\frac{p}{p-q}}\left(H^{-}(x)\right)^{\frac{p q}{p-q}} d x\right)^{\frac{p-q}{p q}} .
\end{aligned}
$$

Theorem 2.1. Let $0<q<p, p \geq 1$ and $0<\theta<\infty$. If $E^{ \pm}<\infty$, then inequality (1.1) holds. Moreover, $C^{ \pm} \ll E^{ \pm}$, where $C^{ \pm}>0$ is the best constant in (1.1).

Proof. Let us prove Theorem 2.1 only for the operator $T_{\theta}^{+}$. For the operator $T_{\theta}^{-}$ it can be proved similarly.

In the same way as in the proof of the sufficiency part of Theorem 2.1 of [5] we define a sequence of points $\left\{x_{k}\right\} \subset I$ such that

$$
\begin{gathered}
I=\bigcup_{k}\left[x_{k}, x_{k+1}\right), \quad\left[x_{k}, x_{k+1}\right) \bigcap\left[x_{i}, x_{i+1}\right)=\varnothing, \quad i \neq k, \\
\left(T_{\theta}^{+} f\left(x_{k}\right)\right)^{\theta} \equiv \int_{0}^{x_{k}} w(t)\left(\int_{t}^{x_{k}} f(s) d s\right)^{\theta} d t=2^{\theta k} \text { if } x_{k}<\infty, \\
2^{\theta k} \leq\left(T_{\theta}^{+} f(x)\right)^{\theta} \equiv \int_{0}^{x} w(t)\left(\int_{t}^{x} f(s) d s\right)^{\theta} d t<2^{\theta(k+1)} \text { if } x_{k} \leq x<x_{k+1} .
\end{gathered}
$$

From (2.2) and (2.3) it follows

$$
2^{k-1} \ll\left(\int_{x_{k-1}}^{x_{k}} w(t)\left(\int_{t}^{x_{k}} f(s) d s\right)^{\theta} d t\right)^{\frac{1}{\theta}}+\left(\int_{0}^{x_{k-1}} w(t) d t\right)_{x_{k-1}}^{\frac{1}{\theta}} \int_{x_{k}}^{x_{k}} f(s) d s .
$$

Using (2.1), (2.2) and (2.4) as in [5] we have

$$
\begin{aligned}
L \equiv \int_{0}^{\infty} u(x)\left(T_{\theta}^{+} f(x)\right)^{q} d x=\sum_{k} \int_{x_{k}}^{x_{k+1}} u(x)\left(T_{\theta}^{+} f(x)\right)^{q} d x \leq 2^{2 q} \sum_{k} 2^{q(k-1)} \int_{x_{k}}^{x_{k+1}} u(x) d x \\
\ll \sum_{k}\left(\int_{x_{k-1}}^{x_{k}} w(t)\left(\int_{t}^{x_{k}} f(s) d s\right)^{\theta} d t\right)^{\frac{q}{\theta}} \int_{x_{k}}^{x_{k+1}} u(x) d x \\
+\sum_{k}\left(\int_{0}^{x_{k-1}} w(t) d t\right)^{\frac{q}{\theta}}\left(\int_{x_{k-1}}^{x_{k}} f(s) d s\right)^{q} \int_{x_{k}}^{x_{k+1}} u(x) d x=L_{1}+L_{2} . \quad(2.5)
\end{aligned}
$$


Let us estimate $L_{1}$ and $L_{2}$ separately.

To estimate $L_{1}$ first we use Hardy inequality, then we apply Hölder's inequality for sequences with the parameters $\frac{p}{q}$ and $\frac{p}{p-q}$ and get

$$
\begin{gathered}
L_{1} \leq \sum_{k} \int_{x_{k}}^{x_{k+1}} u(x) d x\left(H^{+}\left(x_{k-1}, x_{k}\right)\right)^{q}\left(\int_{x_{k-1}}^{x_{k}} v(t) f^{p}(t) d t\right)^{\frac{q}{p}} \\
\leq\left(\sum_{k}\left(\int_{x_{k}}^{x_{k+1}} u(x) d x\right)^{\frac{p}{p-q}}\left(H^{+}\left(x_{k-1}, x_{k}\right)\right)^{\frac{q p}{p-q}}\right)^{\frac{p-q}{p}}\left(\sum_{k} \int_{x_{k-1}}^{x_{k}} v(t) f^{p}(t) d t\right)^{\frac{q}{p}} \\
\ll\left(\sum_{k} \int_{x_{k}}^{x_{k+1}} u(x)\left(\int_{x}^{x_{k+1}} u(s) d s\right)^{\frac{q}{p-q}}\left(H^{+}\left(x_{k-1}, x_{k}\right)\right)^{\frac{q p}{p-q}} d x\right)^{\frac{p-q}{p}}\left(\int_{0}^{\infty} v(t) f^{p}(t) d t\right)^{\frac{q}{p}} \\
\ll\left(\sum_{k} \int_{x_{k}}^{x_{k+1}} u(x)\left(\int_{x}^{\infty} u(s) d s\right)^{\frac{q}{p-q}}\left(H^{+}(0, x)\right)^{\frac{q p}{p-q}} d x\right)^{\frac{p-q}{p}}\left(\int_{0}^{\infty} v(t) f^{p}(t) d t\right)^{\frac{q}{p}} \\
\end{gathered}
$$

Now, we estimate $L_{2}$ for each case of Lemma A separately.

Let $1 \leq p \leq \theta<\infty$. Twice using Hölder's inequality we get

$$
\begin{gathered}
L_{2} \leq \sum_{k}\left(\int_{0}^{x_{k-1}} w(t) d t\right)^{\frac{q}{\theta}}\left(\int_{x_{k-1}}^{x_{k}} v^{1-p^{\prime}}(s) d s\right)^{\frac{q}{p^{\prime}}} \int_{x_{k}}^{x_{k+1}} u(x) d x\left(\int_{x_{k-1}}^{x_{k}} v(t) f^{p}(t) d t\right)^{\frac{q}{p}} \\
\leq\left(\sum_{k}\left(\int_{x_{k}}^{x_{k+1}} u(x) d x\right)^{\frac{p}{p-q}}\left(\left(\int_{0}^{x_{k-1}} w(t) d t\right)^{\frac{1}{\theta}}\left(\int_{x_{k-1}}^{x_{k}} v^{1-p^{\prime}}(s) d s\right)^{\frac{1}{p^{\prime}}}\right)^{\frac{q p}{p-q}}\right)^{\frac{p-q}{p}} \\
\times\left(\sum_{k} \int_{x_{k-1}}^{x_{k}} v(t) f^{p}(t) d t\right)^{\frac{q}{p}}
\end{gathered}
$$




$$
\begin{aligned}
& \ll\left(\sum_{k} \int_{x_{k}}^{x_{k+1}} u(x)\left(\int_{x}^{x_{k+1}} u(s) d s\right)^{\frac{q}{p-q}} d x\right. \\
& \times\left(\sup _{0<z<x_{k}}\left(\int_{0}^{z} w(t) d t\right)^{\frac{1}{\theta}}\left(\int_{z}^{x_{k}} v^{1-p^{\prime}}(s) d s\right)^{\frac{1}{p^{\prime}}}\right)^{\frac{q p}{p-q}}\left(\int_{0}^{\frac{p-q}{p}} v(t) f^{p}(t) d t\right)^{\frac{q}{p}} \\
& \leq\left(\sum_{k} \int_{x_{k}}^{x_{k+1}} u(x)\left(\int_{x}^{\infty} u(s) d s\right)^{\frac{q}{p-q}}\left(A^{+}(x)\right)^{\frac{q p}{p-q}} d x\right)^{\frac{p-q}{p}}\left(\int_{0}^{\infty} v(t) f^{p}(t) d t\right)^{\frac{q}{p}}
\end{aligned}
$$

(due to (1.2))

$$
\begin{gathered}
\ll\left(\int_{0}^{\infty} u(x)\left(\int_{x}^{\infty} u(s) d s\right)^{\frac{q}{p-q}}\left(H^{+}(x)\right)^{\frac{q p}{p-q}} d x\right)^{\frac{p-q}{p}}\left(\int_{0}^{\infty} v(t) f^{p}(t) d t\right)^{\frac{q}{p}} \\
=\left(E^{+}\right)^{q}\left(\int_{0}^{\infty} v(t) f^{p}(t) d t\right)^{\frac{q}{p}}
\end{gathered}
$$

Now, let $0<\theta<p$ and $1<p<\infty$. Starting from (2.7) and using Lemma $\mathrm{A}(\mathrm{ii})$, we get

$$
\begin{gathered}
L_{2} \leq\left(\sum_{k}\left(\int_{x_{k}}^{x_{k+1}} u(x) d x\right)^{\frac{p}{p-q}}\left(\int_{0}^{x_{k-1}} w(t) d t\right)^{\frac{q p}{\theta(p-q)}}\right. \\
\left.\times\left(\int_{x_{k-1}}^{x_{k}} v^{1-p^{\prime}}(s) d s\right)^{\frac{q(p-1)}{p-q}}\right)^{\frac{p-q}{p}}\left(\int_{0}^{\infty} v(t) f^{p}(t) d t\right)^{\frac{q}{p}} \\
\ll\left(\sum _ { k } ( \int _ { x _ { k } } ^ { x _ { k + 1 } } u ( x ) d x ) ^ { \frac { p } { p - q } } \left(\int_{0}^{x_{k-1}} w(t)\left(\int_{0}^{t} w(s) d s\right)^{\frac{\theta}{p-\theta}} d t\right.\right. \\
\left.\left.\times\left(\int_{x_{k-1}}^{x_{k}} v^{1-p^{\prime}}(s) d s\right)^{\frac{\theta(p-1)}{p-\theta}}\right)^{\frac{q(p-\theta)}{\theta(p-q)}}\right)^{\frac{p-q}{p}}\left(\int_{0}^{\infty} v(t) f^{p}(t) d t\right)^{\frac{q}{p}}
\end{gathered}
$$




$$
\begin{gathered}
\leq\left(\sum_{k}\left(\int_{x_{k}}^{x_{k+1}} u(x) d x\right)^{\frac{p}{p-q}}\left(B^{+}\left(x_{k}\right)\right)^{\frac{p q}{p-q}}\right)^{\frac{p-q}{p}}\left(\int_{0}^{\infty} v(t) f^{p}(t) d t\right)^{\frac{q}{p}} \\
\ll\left(\sum_{k} \int_{x_{k}}^{x_{k+1}} u(x)\left(\int_{x}^{\infty} u(t) d t\right)^{\frac{q}{p-q}}\left(H^{+}(x)\right)^{\frac{p q}{p-q}} d x\right)^{\frac{p-q}{p}}\left(\int_{0}^{\infty} v(t) f^{p}(t) d t\right)^{\frac{q}{p}} \\
\leq\left(E^{+}\right)^{q}\left(\int_{0}^{\infty} v(t) f^{p}(t) d t\right)^{\frac{q}{p}}
\end{gathered}
$$

In the case $0<\theta<1=p$ we have, following (2.5),

$$
\begin{gathered}
L_{2}=\sum_{k}\left(\int_{0}^{x_{k-1}} w(t) d t\right)^{\frac{q}{\theta}}\left(\int_{x_{k-1}}^{x_{k}} \frac{1}{v(t)} v(t) f(t) d t\right)^{q} \int_{x_{k}}^{x_{k+1}} u(x) d x \\
\leq \sum_{k} \int_{x_{k}}^{x_{k+1}} u(x) d x\left(\int_{0}^{x_{k-1}} w(t) d t\right)^{\frac{q}{\theta}}\left(\underline{v}\left(x_{k-1}, x_{k}\right)\right)^{-q}\left(\int_{x_{k-1}}^{x_{k}} v(t) f(t) d t\right)^{q}
\end{gathered}
$$

(since we have that $q<p=1$, we use Hölder's inequality with the parameters $\frac{1}{q}$ and $\frac{1}{1-q}$ )

$$
\begin{aligned}
& \leq\left(\sum_{k}\left(\int_{x_{k}}^{x_{k+1}} u(x) d x\right)^{\frac{1}{1-q}}\left(\int_{0}^{x_{k-1}} w(t) d t\right)^{\frac{q}{\theta(1-q)}}\left(\underline{v}\left(x_{k-1}, x_{k}\right)\right)^{\frac{q}{q-1}}\right)^{1-q} \\
& =\left(\sum _ { k } ( \int _ { x _ { k } } ^ { x _ { k + 1 } } u ( x ) d x ) ^ { \frac { 1 } { 1 - q } } \left(\left(\int_{k}^{x_{x_{k-1}}} \int^{x_{k}} v(t) f(t) d t\right)^{q}\right.\right. \\
& \\
& =\left(\int_{0}^{\frac{1}{1-\theta}}(t) d t\right)^{\left.\left.\left.\frac{q}{v}\left(x_{k-1}, x_{k}\right)\right)^{\frac{\theta}{\theta-1}}\right)^{\frac{q(1-\theta)}{\theta(1-q)}}\right)^{1-q}} \\
& \times\left(\int_{0}^{\infty} v(t) f(t) d t\right)^{q}
\end{aligned}
$$




$$
\begin{gathered}
\qquad\left(\sum_{k}\left(\int_{x_{k}}^{x_{k+1}} u(x) d x\right)^{\frac{1}{1-q}}\left(\int_{0}^{x_{k-1}} w(t)\left(\int_{0}^{t} w(s) d s\right)^{\frac{\theta}{1-\theta}}\left(\underline{v}\left(t, x_{k}\right)\right)^{\frac{\theta}{\theta-1}} d t\right)^{\frac{q(1-\theta)}{\theta(1-q)}}\right)^{1-q}\left(\int_{0}^{\infty} v(t) f(t) d t\right)^{q} \\
\ll\left(\sum_{k} \int_{x_{k}}^{x_{k+1}} u(x)\left(\int_{x}^{x_{k+1}} u(s) d s\right)^{\frac{q}{1-q}}\left(D^{+}(x)\right)^{\frac{q}{1-q}} d x\right)^{1-q}\left(\int_{0}^{\infty} v(t) f(t) d t\right)^{q} \\
\ll\left(\int_{0}^{\infty} u(x)\left(\int_{x}^{\infty} u(s) d s\right)^{\frac{q}{1-q}}\left(H^{+}(x)\right)^{\frac{q}{1-q}} d x\right)^{1-q}\left(\int_{0}^{\infty} v(t) f(t) d t\right)^{\frac{q}{p}} \\
\\
\ll
\end{gathered}
$$

From (2.5), (2.6), (2.8), (2.9) and (2.10) it follows that (1.1) holds with the estimate $C^{+} \ll E^{+}$for the best constant $C^{+}>0$ in (1.1). The proof of Theorem 2.1 is complete.

Let

$$
\begin{aligned}
& F^{+}=\left(\int_{0}^{\infty} u(x)\left(\int_{x}^{\infty} u(s) d s\right)^{\frac{q}{p-q}}\left(B^{+}(x)\right)^{\frac{p q}{p-q}} d x\right)^{\frac{p-q}{p q}} \\
& F^{-}=\left(\int_{0}^{\infty} u(x)\left(\int_{0}^{x} u(s) d s\right)^{\frac{p}{p-q}}\left(B^{-}(x)\right)^{\frac{p q}{p-q}} d x\right)^{\frac{p-q}{p q}}
\end{aligned}
$$

Theorem 2.2. Let $0<\theta, q<\infty, \max \{\theta, q\}<p$ and $p>1$. Then inequality (1.1) holds if and only if $E^{ \pm}<\infty$. Moreover, $E^{ \pm} \approx C^{ \pm}$, where $C^{ \pm}>0$ is the best constant in (1.1).

Proof. The sufficiency follows from Theorem 2.1.

We prove the necessity for the operator $T_{\theta}^{+}$. For the operator $T_{\theta}^{-}$it can be proved analogously. Suppose that inequality (1.1) holds for $T_{\theta}^{+}$with the best constant $C^{+}>0$. It suffices to prove that $F^{+} \ll C^{+}$since in the case $\max \{\theta, q\}<$ $p$ and $p>1$ we have that $F^{ \pm} \approx E^{ \pm}$, by Lemma A. We consider two cases $q \leq \theta$ and $q>\theta$. 
First we consider the case $q \leq \theta$. Let $0<y<z<\infty$. Due to local integrability of the functions $w$ and $v^{1-p^{\prime}}$ on $I$ the following function

$$
\begin{aligned}
F(x) \equiv F_{y}(x)=\int_{y}^{x} w(t)\left(\int_{y}^{t} w(s) d s\right)^{\frac{\theta}{p-\theta}}\left(\int_{t}^{x} v^{1-p^{\prime}}(s) d s\right)^{\frac{\theta(p-1)}{p-\theta}} d t \\
=\left(B^{+}(y, x)\right)^{\frac{p \theta}{p-\theta}}
\end{aligned}
$$

is defined for all $x>y$.

The function $F(x)$ for any $\tau>y$ is absolutely continuous on the interval $[y, \tau]$. Therefore, its derivative

$$
\begin{array}{r}
F^{\prime}(x)=\frac{\theta(p-1)}{p-\theta} \int_{y}^{x} w(t)\left(\int_{y}^{t} w(s) d s\right)^{\frac{\theta}{p-\theta}}\left(\int_{t}^{x} v^{1-p^{\prime}}(s) d s\right)^{\frac{p(\theta-1)}{p-\theta}} d t v^{1-p^{\prime}}(x) \\
\equiv \frac{\theta(p-1)}{p-\theta} g(x) v^{1-p^{\prime}}(x)
\end{array}
$$

is integrable on the interval $[y, \tau]$ for any $\tau>y$. Here

$$
g(x)=\int_{y}^{x} w(t)\left(\int_{y}^{t} w(s) d s\right)^{\frac{\theta}{p-\theta}}\left(\int_{t}^{x} v^{1-p^{\prime}}(s) d s\right)^{\frac{p(\theta-1)}{p-\theta}} d t .
$$

We introduce the function

$$
f_{y, z}(t)=\chi_{(y, z)}(t)\left(\int_{t}^{z} u(x) d x\right)^{\frac{1}{p-q}}(F(t))^{\frac{q-\theta}{\theta(p-q)}}(g(t))^{\frac{1}{p}} v^{1-p^{\prime}}(t)
$$

where $\chi_{(y, z)}(\cdot)$ is the characteristic function of the interval $(y, z)$. Then due to local integrability of the functions $u, w, v^{1-p^{\prime}}$ and $g v^{1-p^{\prime}}$ we have

$$
\left(\int_{0}^{\infty} v(t) f_{y, z}^{p}(t) d t\right)^{\frac{1}{p}}=\left(\int_{y}^{z}\left(\int_{t}^{z} u(x) d x\right)^{\frac{p}{p-q}}(F(t))^{\frac{p(q-\theta)}{\theta(p-q)}} g(t) v^{1-p^{\prime}}(t) d t\right)^{\frac{1}{p}}<\infty .
$$

From the last expression by integration by parts we get

$$
\left(\int_{0}^{\infty} v(t) f_{y, z}^{p}(t) d t\right)^{\frac{1}{p}} \approx\left(\int_{y}^{z} u(t)\left(\int_{t}^{z} u(x) d x\right)^{\frac{q}{p-q}}(F(t))^{\frac{q(p-\theta)}{\theta(p-q)}} d t\right)^{\frac{1}{p}} .
$$

We estimate the left side of (1.1) for $f=f_{y, z}$ from below. For this purpose first we estimate the expression $T_{\theta}^{+} f_{y, z}(x)$ for a fixed $x \in(y, z)$ from below. Using monotonicity of the functions $(F(t))^{\frac{q-\theta}{\theta(p-q)}}$ and $\left(\int_{t}^{z} u(x) d x\right)^{\frac{1}{p-q}}$ for $t \in(y, z)$ we 
have

$$
\begin{gathered}
\left(T_{\theta}^{+} f_{y, z}(x)\right)^{\theta}=\int_{y}^{x} w(t)\left(\int_{t}^{x} f_{y, z}(s) d s\right)^{\theta} d t \\
=\int_{y}^{x} w(t)\left(\int_{t}^{x}\left(\int_{s}^{z} u(t) d t\right)^{\frac{1}{p-q}}(F(s))^{\frac{q-\theta}{\theta(p-q)}} g^{\frac{1}{p}}(s) v^{1-p^{\prime}}(s) d s\right)^{\theta} d t \\
\geq\left(\int_{x}^{z} u(t) d t\right)^{\frac{\theta}{p-q}}(F(x))^{\frac{q-\theta}{p-q}} \int_{y}^{x} w(t)\left(\int_{t}^{x} g^{\frac{1}{p}}(s) v^{1-p^{\prime}}(s) d s\right)^{\theta} d t .
\end{gathered}
$$

We estimate the integral $\int_{t}^{x} g^{\frac{1}{p}}(s) v^{1-p^{\prime}}(s) d s$ separately:

$$
\begin{gathered}
\int_{t}^{x} g^{\frac{1}{p}}(s) v^{1-p^{\prime}}(s) d s \\
=\int_{t}\left(\int_{y}^{s} w(\varsigma)\left(\int_{y}^{\varsigma} w(\tau) d \tau\right)^{\frac{\theta}{p-\theta}}\left(\int_{\varsigma}^{s} v^{1-p^{\prime}}(\tau) d \tau\right)^{\frac{p(\theta-1)}{p-\theta}} d \varsigma\right)^{\frac{1}{p}} v^{1-p^{\prime}}(s) d s \\
\left.\geq \int_{t}^{x} \int_{y}^{t} w(\varsigma)\left(\int_{y}^{\varsigma} w(\tau) d \tau\right)^{\frac{\theta}{p-\theta}}\left(\int_{t}^{s} v^{1-p^{\prime}}(\tau) d \tau\right)^{\frac{p(\theta-1)}{p-\theta}} d \varsigma\right)^{\frac{1}{p}} v^{1-p^{\prime}}(s) d s \\
\approx\left(\int_{y}^{t} w(\tau) d \tau\right)^{\frac{1}{p-\theta}} \int_{t}^{x}\left(\int_{t}^{s} v^{1-p^{\prime}}(\tau) d \tau\right)^{\frac{\theta-1}{p-\theta}} v^{1-p^{\prime}}(s) d s \\
\approx\left(\int_{y}^{t} w(\tau) d \tau\right)^{\frac{1}{p-\theta}}\left(\int_{t}^{x} v^{1-p^{\prime}}(\tau) d \tau\right)^{\frac{p-1}{p-\theta}} \cdot
\end{gathered}
$$

From (2.12) and (2.13) for $x \in(y, z)$ we have

$$
\begin{aligned}
T_{\theta}^{+} f_{y, z}(x) \gg & \left(\int_{x}^{z} u(t) d t\right)^{\frac{1}{p-q}}(F(x))^{\frac{q-\theta}{\theta(p-q)}} \\
& \left(\int_{y}^{x} w(t)\left(\int_{y}^{t} w(\tau) d \tau\right)^{\frac{\theta}{p-\theta}}\left(\int_{t}^{x} v^{1-p^{\prime}}(\tau) d \tau\right)^{\frac{\theta(p-1)}{p-\theta}} d t\right)^{\frac{1}{\theta}}
\end{aligned}
$$




$$
=\left(\int_{x}^{z} u(t) d t\right)^{\frac{1}{p-q}}(F(x))^{\frac{p-\theta}{\theta(p-q)}} .
$$

Then

$$
\begin{gathered}
\left(\int_{0}^{\infty} u(x)\left(T_{\theta}^{+} f_{y, z}(x)\right)^{q} d x\right)^{\frac{1}{q}} \geq\left(\int_{y}^{z} u(x)\left(T_{\theta}^{+} f_{y, z}(x)\right)^{q} d x\right)^{\frac{1}{q}} \\
\gg\left(\int_{y}^{z} u(x)\left(\int_{x}^{z} u(t) d t\right)^{\frac{q}{p-q}}(F(x))^{\frac{q(p-\theta)}{\theta(p-q)}} d x\right)^{\frac{1}{q}} .
\end{gathered}
$$

From (1.1), (2.11) and (2.14) we get

$$
\left(\int_{y}^{z} u(x)\left(\int_{x}^{z} u(t) d t\right)^{\frac{q}{p-q}}(F(x))^{\frac{q(p-\theta)}{\theta(p-q)}} d x\right)^{\frac{p-q}{p q}} \ll C^{+}
$$

for all $(y, z) \subset I$.

Proceeding to the limits $y \rightarrow 0$ and $z \rightarrow \infty$ and taking into account that $\lim _{y \rightarrow 0} F_{y, z}(x)=\left(B^{+}(x)\right)^{\frac{p \theta}{p-\theta}}$ we have $z \rightarrow \infty$

$$
F^{+} \ll C^{+} \text {. }
$$

Thus, the proof of the necessity for the case $q \leq \theta$ is complete.

Now, let $q>\theta$. Then $\gamma=\frac{q}{\theta}>1$. Let $f$ and $\varphi$ be non-negative functions such that $\int_{0}^{\infty} v(t) f^{p}(t) d t<\infty$ and $\int_{0}^{\infty} u^{1-\gamma^{\prime}}(s) \varphi^{\gamma^{\prime}}(s) d s<\infty$. Inequality (1.1) is rewritten in the form:

$$
\left(\int_{0}^{\infty} u(x)\left(\int_{0}^{x} w(t)\left(\int_{t}^{x} f(s) d s\right)^{\theta} d t\right)^{\gamma} d x\right)^{\frac{1}{\gamma}} \leq\left(C^{+}\right)^{\theta}\left(\int_{0}^{\infty} v(t) f^{p}(t) d t\right)^{\frac{\theta}{p}} .
$$

By Hölder's inequality, this implies

$$
\begin{aligned}
& \int_{0}^{\infty} \varphi(x) \int_{0}^{x} w(t)\left(\int_{t}^{x} f(s) d s\right)^{\theta} d t d x \\
& \leq\left(C^{+}\right)^{\theta}\left(\int_{0}^{\infty} u^{1-\gamma^{\prime}}(s) \varphi^{\gamma^{\prime}}(s) d s\right)^{\frac{1}{\gamma^{\prime}}}\left(\int_{0}^{\infty} v(t) f^{p}(t) d t\right)^{\frac{\theta}{p}} .
\end{aligned}
$$


Since $f$ was chosen arbitrarily, we get

$$
G \equiv \sup _{f \geq 0} \frac{\left(\int_{0}^{\infty} \varphi(x) \int_{0}^{x} w(t)\left(\int_{t}^{x} f(s) d s\right)^{\theta} d t d x\right)^{\frac{1}{\theta}}}{\left(\int_{0}^{\infty} v(t) f^{p}(t) d t\right)^{\frac{1}{p}}} \leq C^{+}\left(\int_{0}^{\infty} u^{1-\gamma^{\prime}}(s) \varphi^{\gamma^{\prime}}(s) d s\right)^{\frac{1}{\theta \gamma^{\prime}}}
$$

For the fixed $\varphi$, the quantity $G$ in $(2.16)$ is equal to the least constant $C^{+}$of inequality (1.1) in which $u(x) \equiv \varphi(x)$ and $q=\theta$.

Therefore, using the first part of the proof, we have

$$
G \gg\left(\int_{0}^{\infty} \varphi(x)\left(\int_{x}^{\infty} \varphi(t) d t\right)^{\frac{\theta}{p-\theta}} \widetilde{F}(x) d x\right)^{\frac{p-\theta}{\theta p}}
$$

where $\widetilde{F}(x)=\left(B^{+}(x)\right)^{\frac{p \theta}{p-\theta}}$.

Integration by parts of the last expression gives

$$
G \gg\left(\int_{0}^{\infty}\left(\int_{x}^{\infty} \varphi(t) d t\right)^{\frac{p}{p-\theta}} \widetilde{F}^{\prime}(x) d x\right)^{\frac{p-\theta}{\theta p}}
$$

Then from (2.16) we have the following Hardy inequality:

$$
\left(\int_{0}^{\infty}\left(\int_{x}^{\infty} \varphi(t) d t\right)^{\mu} \tilde{F}^{\prime}(x) d x\right)^{\frac{1}{\mu}} \ll\left(C^{+}\right)^{\theta}\left(\int_{0}^{\infty} u^{1-\gamma^{\prime}}(s) \varphi^{\gamma^{\prime}}(s) d s\right)^{\frac{1}{\gamma^{\prime}}}
$$

where $\mu=\frac{p}{p-\theta}$.

Since $\gamma^{\prime}=\frac{q}{q-\theta}$, it holds $\gamma^{\prime}>\mu$. Since $\varphi$ was arbitrary, (2.17) holds for all $\varphi$ such that $\int_{0}^{\infty} u^{1-\gamma^{\prime}}(s) \varphi^{\gamma^{\prime}}(s) d s<\infty$. Hence, by Lemma A we have

$$
\left(\int_{0}^{\infty} \widetilde{F}^{\prime}(x)\left(\int_{0}^{x} \widetilde{F}^{\prime}(t) d t\right)^{\frac{\mu}{\gamma^{\prime}-\mu}}\left(\int_{x}^{\infty} u(s) d s\right)^{\frac{\mu\left(\gamma^{\prime}-1\right)}{\gamma^{\prime}-\mu}} d x\right)^{\frac{\gamma^{\prime}-\mu}{\mu \gamma^{\prime}}} \ll\left(C^{+}\right)^{\theta} .
$$

Integration by parts yields

$$
\left(\int_{0}^{\infty} u(x)\left(\int_{x}^{\infty} u(s) d s\right)^{\frac{\gamma^{\prime}(\mu-1)}{\gamma^{\prime}-\mu}}(\widetilde{F}(x))^{\frac{\gamma^{\prime}}{\gamma^{\prime}-\mu}} d x\right)^{\frac{\gamma^{\prime}-\mu}{\theta \mu \gamma^{\prime}}} \ll C^{+} .
$$


Since $\frac{\gamma^{\prime}(\mu-1)}{\gamma^{\prime}-\mu}=\frac{q}{p-q}, \frac{\gamma^{\prime}}{\gamma^{\prime}-\mu}=\frac{q(p-\theta)}{\theta(p-q)}, \frac{\gamma^{\prime}-\mu}{\theta \mu \gamma^{\prime}}=\frac{p-q}{p q}$ and $(\widetilde{F}(x))^{\frac{p-\theta}{p \theta}}=B^{+}(x)$, we have

$$
F^{+} \ll C^{+} \text {. }
$$

Relations (2.15) and (2.18), together with the relation $C^{+} \ll E^{+}$obtained in Theorem 2.1, give $E^{+} \approx C^{+}$. The proof of Theorem 2.2 is complete.

Let

$$
\begin{aligned}
& F_{1}^{+}=\left(\int_{0}^{\infty} u(x)\left(\int_{x}^{\infty} u(s) d s\right)^{\frac{q}{1-q}}\left(\int_{0}^{x} w(s) d s\right)^{\frac{q}{\theta(1-q)}} d x\right)^{\frac{1-q}{q}}, \\
& F_{1}^{-}=\left(\int_{0}^{\infty} u(x)\left(\int_{0}^{x} u(s) d s\right)^{\frac{q}{1-q}}\left(\int_{x}^{\infty} w(s) d s\right)^{\frac{q}{\theta(1-q)}} d x\right)^{\frac{1-q}{q}} .
\end{aligned}
$$

Theorem 2.3. Let $\max \{\theta, q\}<1=p$ and $v(x) \equiv 1$. Then inequality (1.1) holds if and only if $E^{ \pm}<\infty$. Moreover, $E^{ \pm} \approx C^{ \pm}$, where $C^{ \pm}>0$ is the best constant in (1.1).

Proof. The sufficiency follows from Theorem 2.1.

Let us prove the necessity only for $T_{\theta}^{+}$since for $T_{\theta}^{-}$it can be proved similarly. Suppose that inequality (1.1) holds for $T_{\theta}^{+}$with the best constant $C^{+}>0$. Since $v(x) \equiv 1$, we have $D^{+}(x) \approx\left(\int_{0}^{x} w(s) d s\right)^{\frac{1}{\theta}}$. Here and below the equivalence constants do not depend on $x \in I$. Due to the relations $D^{+}(x) \approx H^{+}(x)$ the values $F_{1}^{+}$are equivalent to the values $E^{+}$, respectively. Therefore, it suffices to prove the estimates $F_{1}^{+} \ll C^{+}$.

Let $0<y<z<\infty$. Assume

$$
f_{y, z}(t)=\chi_{(y, z)}(t)\left(\int_{t}^{z} u(s) d s\right)^{\frac{1}{1-q}}\left(\int_{y}^{t} w(s) d s\right)^{\frac{q}{\theta(1-q)}-1} w(t) .
$$

Then

$$
\begin{aligned}
& \int_{0}^{\infty} f_{y, z}(t) d t=\int_{y}^{z}\left(\int_{t}^{z} u(s) d s\right)^{\frac{1}{1-q}}\left(\int_{y}^{t} w(s) d s\right)^{\frac{q}{\theta(1-q)}-1} w(t) d t \\
& \approx \int_{y}^{z} u(t)\left(\int_{t}^{z} u(s) d s\right)^{\frac{q}{1-q}}\left(\int_{y}^{t} w(s) d s\right)^{\frac{q}{\theta(1-q)}} d t
\end{aligned}
$$


Now, we estimate the left side of (1.1) for $f=f_{y, z}$ from below. Let the function $\sigma(x) \equiv \sigma_{y}(x)$ be such that $\sigma(x)<x$ and

$$
\int_{y}^{x} w(t) d t=2 \int_{y}^{\sigma(x)} w(t) d t \text { for all } x \in(y, \infty) .
$$

Then

$$
\begin{aligned}
& \left(\int_{y}^{x} w(t)\left(\left(\int_{y}^{x} w(s) d s\right)^{\frac{q}{\theta(1-q)}}-\left(\int_{y}^{t} w(s) d s\right)^{\frac{q}{\theta(1-q)}}\right)^{\theta} d t\right)^{\frac{q}{\theta}} \\
\geq & \left(\int_{y}^{\sigma(x)} w(t)\left(\left(\int_{y}^{x} w(s) d s\right)^{\frac{q}{\theta(1-q)}}-\left(\int_{y}^{t} w(s) d s\right)^{\frac{q}{\theta(1-q)}}\right)^{\theta} d t\right)^{\frac{q}{\theta}} \\
\geq & \left(\int_{y}^{\sigma(x)} w(t) d t\right)^{\frac{q}{\theta}}\left(\left(\int_{y}^{x} w(s) d s\right)^{\frac{q}{\theta(1-q)}}-\left(\int_{y}^{\sigma(x)} w(s) d s\right)^{\frac{q}{\theta(1-q)}}\right)^{q} \\
= & \left(\frac{1}{2}\right)^{\frac{q}{\theta}}\left(1-\left(\frac{1}{2}\right)^{\frac{q}{\theta(1-q)}}\right)\left(\int_{y}^{x} w(s) d s\right)^{\frac{q}{\theta(1-q)}} .
\end{aligned}
$$

Using estimate (2.20) for $x \in(y, z)$ we get

$$
\begin{gathered}
\left(T_{\theta}^{+} f_{y, z}(x)\right)^{q}=\left(\int_{y}^{x} w(t)\left(\int_{t}^{x} f_{y, z}(s) d s\right)^{\theta} d t\right)^{\frac{q}{\theta}} \\
\geq\left(\int_{x}^{z} u(s) d s\right)^{\frac{q}{1-q}}\left(\int_{y}^{x} w(t)\left(\int_{t}^{x}\left(\int_{y}^{s} w(\tau) d \tau\right)^{\frac{q}{\theta(1-q)}-1} w(s) d s\right)^{\theta} d t\right)^{\frac{q}{\theta}} \\
\gg\left(\int_{x}^{z} u(s) d s\right)^{\frac{q}{1-q}}\left(\int_{y}^{x} w(t) d t\right)^{\frac{q}{\theta(1-q)}} .
\end{gathered}
$$

Then

$$
\begin{aligned}
& \int_{0}^{\infty} u(x)\left(T_{\theta}^{+} f_{y, z}(x)\right)^{q} d x \geq \int_{y}^{z} u(x)\left(T_{\theta}^{+} f_{y, z}(x)\right)^{q} d x \\
& \gg \int_{y}^{z} u(x)\left(\int_{x}^{z} u(s) d s\right)^{\frac{q}{1-q}}\left(\int_{y}^{x} w(t) d t\right)^{\frac{q}{\theta(1-q)}} d x .
\end{aligned}
$$


From (1.1), (2.19) and (2.22) we have

$$
\left(\int_{y}^{z} u(x)\left(\int_{x}^{z} u(s) d s\right)^{\frac{q}{1-q}}\left(\int_{y}^{x} w(t) d t\right)^{\frac{q}{\theta(1-q)}} d x\right)^{\frac{1-q}{q}} \ll C^{+}
$$

for all $(y, z) \subset I$.

Taking the limits $y \rightarrow 0$ and $z \rightarrow \infty$ we get the estimate $F_{1}^{+} \ll C^{+}$which, together with the estimate $E^{+} \ll C^{+}$from the sufficiency part, gives $E^{+} \approx C^{+}$. The proof of Theorem 2.3 is complete.

\section{Applications}

In the paper [3] the following inequalities

$$
\left\|\varphi \widetilde{H}_{n} f\right\|_{L M_{\theta q, \tau}} \leq C\|f\|_{L_{p, V}}
$$

and

$$
\left\|\varphi H_{n} f\right\|_{\mathcal{C}_{L M_{\theta q, \tau}}} \leq C\|f\|_{L_{p, V}}
$$

were studied, where $L M_{\theta q, \tau}$ is the local Morrey-type space with the norm

$$
\|f\|_{L M_{\theta q, \tau}}=\|\tau(r)\| f\left\|_{L_{\theta}\left(B_{r}\right)}\right\|_{L_{q}(0, \infty)},
$$

and ${ }^{\mathcal{C}} L M_{\theta q, \tau}$ is the complementary local Morrey-type space with the norm

$$
\|f\|_{\mathcal{C}_{L M_{\theta q, \tau}}}=\|\tau(r)\| f\left\|_{L_{\theta}\left(C B_{r}\right)}\right\|_{L_{q}(0, \infty)}
$$

$B_{r}$ is the open ball in $R^{n}$ centered at 0 with radius $r$ and $C B_{r}$ is the complement of the ball $B_{r}$ in $R^{n}$,

$$
H_{n} f(x)=\int_{B_{|x|}} f(s) d s \text { and } \widetilde{H}_{n} f(x)=\int_{C B_{|x|}} f(s) d s
$$

are multidimensional Hardy operators.

In [3] assuming that $\varphi(x) \equiv \varphi(|x|)$ and $V(x) \equiv V(|x|)$ it was proved that the validity of inequalities (3.1) and (3.2) are equivalent to the validity of the inequalities

$$
\left(\int_{0}^{\infty} u(x)\left(\int_{0}^{x} w(t)\left(\int_{t}^{\infty} f(s) d s\right)^{\theta} d t\right)^{\frac{q}{\theta}} d x\right)^{\frac{1}{q}} \leq C\left(\int_{0}^{\infty} v(t) f^{p}(t) d t\right)^{\frac{1}{p}}
$$

and

$$
\left(\int_{0}^{\infty} u(x)\left(\int_{x}^{\infty} w(t)\left(\int_{0}^{t} f(s) d s\right)^{\theta} d t\right)^{\frac{q}{\theta}} d x\right)^{\frac{1}{q}} \leq C\left(\int_{0}^{\infty} v(t) f^{p}(t) d t\right)^{\frac{1}{p}}
$$

respectively, where $u(x)=\tau^{q}(x), w(t)=\varphi(t) t^{\frac{n-1}{\theta}}$ and $v(t)=V(t) t^{-\frac{n-1}{p^{\prime}}}$.

In the papers [1], [2] and [6] by different approaches necessary and sufficient conditions for the validity of inequalities (3.3) and (3.4) are obtained for different 
relations between the parameters $0<p, q, \theta \leq \infty$. Moreover, in [6] other inequalities of the type (3.3) and (3.4) are considered. In [3] characterizations of (3.3) and (3.4) are found only for the case $1 \leq p \leq q<\infty$ and $0<\theta<\infty$ but by a method different from those in [1], [2] and [6].

Investigation of inequality (1.1) gives this alternative method to characterize inequality (3.3) since the validity of inequality (3.3) is equivalent to the validity of inequality (1.1) for $T_{\theta}^{+}$and the Hardy inequality

$$
\left(\int_{0}^{\infty} u(x)\left(\int_{0}^{x} w(t) d t\right)^{\frac{q}{\theta}}\left(\int_{x}^{\infty} f(s) d s\right)^{q} d x\right)^{\frac{1}{q}} \leq C_{1}\left(\int_{0}^{\infty} v(t) f^{p}(t) d t\right)^{\frac{1}{p}}
$$

The similar splitting can be done for inequality (3.4).

Therefore, for example, from Theorem 2.2 and Lemma A we have

Theorem 3.1. Let $0<\theta, q<\infty, p>1$ and $\max \{\theta, q\}<p$. Let $\varphi(x)=\varphi(|x|)$, $V(x)=V(|x|), u(x)=\tau^{q}(x), w(t)=\varphi(t) t^{\frac{n-1}{\theta}}$ and $v(t)=V(t) t^{-\frac{n-1}{p^{\prime}}}$. Then inequality (3.1) ((3.3)) holds if and only if $E^{+}<\infty$ and

$$
\begin{aligned}
G^{+}=\left(\int_{0}^{\infty} u(x)\left(\int_{0}^{x} w\right)^{\frac{q}{\theta}}\left(\int_{0}^{x} u(t)\left(\int_{0}^{t} w\right)^{\frac{q}{\theta}} d t\right)^{\frac{q}{p-q}}\right. \\
\\
\left.\times\left(\int_{x}^{\infty} v^{1-p^{\prime}}\right)^{\frac{q(p-1)}{p-q}} d x\right)^{\frac{p-q}{p q}}<\infty .
\end{aligned}
$$

Moreover, $\max \left\{E^{+}, G^{+}\right\} \approx C$, where $C>0$ is the best constant in (3.1) ((3.3)).

The similar statement follows from Theorem 2.2 and Lemma A for inequality (3.2) ((3.4)).

The characterizations of inequality (3.3) in Theorem 3.1 are respectively equivalent to those obtained earlier in [1](Theorem 3.1, (iv)) and in [6](Theorem 5, $\max \{\theta, q\}<p)$.

Let us also note that inequalities of the type (3.3) and (3.4) with kernels are considered in [7].

Acknowledgements. The paper was written under financial support by the Scientific Committee of the Ministry of Education and Science of Kazakhstan, Grant No.5499/GF4 on priority area "Intellectual potential of the country".

We would like to thank the careful referees for some generous suggestions, which have improved the final version of this paper.

\section{REFERENCES}

1. A. Gogatishvili, R. Mustafayev and L.-E. Persson, Some new iterated Hardy-type inequalities, J. Funct. Spaces Appl. (2012), Article ID 734194. 
2. A. Gogatishvili, R. Mustafayev and L.-E. Persson, Some new iterated Hardy-type inequalities: the case $\theta=1$, J. Inequal. Appl. 2013, 2013:515.

3. A. Kalybay, On boundedness of the conjugate multidimensional Hardy operator from a Lebesgue space to a local Morrey-type space, Int. J. Math. Anal. 8 (2014), no. 11, 539-553.

4. A. Kufner, L. Maligranda and L.-E. Persson, The Hardy inequality. About its history and some related results, Vydavatelský servis, Pilsen, 2007.

5. R. Oinarov and A. Kalybay, Three-parameter weighted Hardy type inequalities, Banach J. Math. Anal. 2 (2008), no. 2, 85-93.

6. D. Prokhorov and V. Stepanov, On weighted Hardy inequalities in mixed norms, Proc. Steklov Inst. Math. 283 (2013), 149-164.

7. D. Prokhorov and V. Stepanov, Weighted estimates for a class of sublinear operators, Doklady Math. 88 (2013), no. 3, 721-723.

8. G. Sinnamon and V. Stepanov, The weighted Hardy inequality: new proofs and case $p=1$, J. London Math. Soc. 54 (1996), 89-101.

${ }^{1}$ L.N. Gumilyov Eurasian National University, Munaytpasov St.5, Astana 010008, KAZAKHSTAN.

E-mail address: o_ryskul@mail.ru; oinarov_r@enu.kz

${ }^{2}$ KIMeP University, Abai Ave.4, Almaty 050010, Kazakhstan.

E-mail address: kalybay@kimep.kz 